\title{
Optimizing informed consent for percutaneous coronary intervention: a mixed methods study
}

\section{Felicity Astin*, \\ J. Probyn, \\ D. Conway, \\ J. Greenhalgh, \\ J. Holt, \\ K. Marshall, \\ J. Wright}

University of Huddersfield Calderdale and Huddersfield NHS Trust, Huddersfield, United Kingdom
KEYWORDS: informed consent, percutaneous coronary intervention. CITATION: Cardiol Croat. 2016;11(10-11):586. | DOI: http://dx.doi.org/10.15836/ccar2016.586 *ADDRESS FOR CORRESPONDENCE: Felicity Astin, Queensgate, Huddersfield HD1 3DH, United Kingdom. Phone: +44 (0) 1484472893 / E-mail: f.astin@hud.ac.uk ORCID: Felicity Astin, http://orcid.org/0000-0002-8055-3072

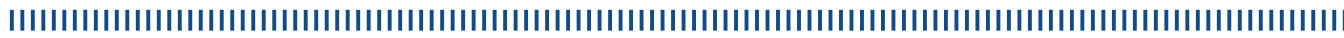
Background: Percutaneous coronary intervention (PCI) is a common treatment to vascularise myocardial muscle. ${ }^{1}$ Serious complication are uncommon, but death is one of them. Doctors must obtain consent before doing PCI; for this to be sound the patient must be mentally able, act of their own free will, and be given enough information to help their decision making. Obtaining valid consent is a legal and ethical requirement. However, published studies tell us that the amount and quality of information given to PCI patients is variable; benefits are often overestimated, risks forgotten and alternative treatments not always considered. Patients may not be informed in the way that they should be.

Patients and Methods: In this mixed methods study, we aimed to describe the PCI informed consent process and survey the attitudes of patients and cardiologists about informed consent in England. We conducted in-depth interviews with 41 patients, 19 cardiologists, and recorded 37 consent conversations at two hospitals. We also sent questionnaires to 326 patients and 124 cardiologists recruited across England to find out their views on consent

Results: Patients were mostly satisfied with the PCI consent process, valued cardiologists as the 'experts', and saw PCI as a 'fix' for their heart condition. The informed consent process was seen as 'paperwork' that was not especially important, but necessary to get access to treatment. Most patients (81\%) received written information before PCI and had (94\%) signed the consent form on the day of treatment. Patients were not very concerned by this; most (81\%) reported being highly satisfied by the explanation they got. The benefits of PCI were overestimated, most (89\%) wanted information about all risks, fewer (84\%) were interested in knowing about alternative treatments or what would happen if they did not have PCI.

Conclusion: Patients and cardiologists were generally satisfied with the PCI consent process, but the reality of practice does not fully mirror practice recommendations in England.
RECEIVED:

September 20, 2016

ACCEPTED:

October 10, 2016

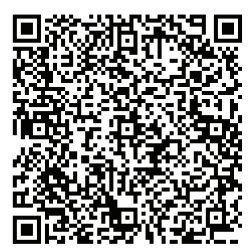

LITERATURE IIIIIIIIIIIIIIIIIIIIIIIIIIIIIIIIIIIIIIIIIIIIIIIIIIIIIIIIIIIIIIIIIIIIIIIIIIIIIIIIIIIIIIIIIIIIIIIIIIII

1. Roldan CA. Valvular and coronary heart disease in systemic inflammatory diseases: Systemic Disorders in heart disease. Heart. 2008:94(8):1089-101.DOI: http://dx.doi.org/10.1136/hrt.2007.132787 